\title{
Sonography and hypotension: a change to critical problem solving in undergraduate medical education
}

\section{Richard Amini \\ Lori A Stolz \\ Nicholas C Hernandez \\ Kevin Gaskin \\ Nicola Baker \\ Arthur Barry Sanders \\ Srikar Adhikari}

Department of Emergency Medicine, University of Arizona Medical Center, College of Medicine, University of Arizona, Tucson, AZ, USA
Correspondence: Richard Amini Department of Emergency Medicine, University of Arizona Medical Center, PO Box 245057, Tucson,

AZ 85724-5057, USA

$\mathrm{Tel}+\mathrm{I} 5206269604$

Fax +I 5206262480

Email richardamini@gmail.com
This article was published in the following Dove Press journal:

Advances in Medical Education and Practice

14 January 2016

Number of times this article has been viewed

Study objectives: Multiple curricula have been designed to teach medical students the basics of ultrasound; however, few focus on critical problem-solving. The objective of this study is to determine whether a theme-based ultrasound teaching session, dedicated to the use of ultrasound in the management of the hypotensive patient, can impact medical students' ultrasound education and provide critical problem-solving exercises.

Methods: This was a cross-sectional study using an innovative approach to train 3rd year medical students during a 1-day ultrasound training session. The students received a 1-hour didactic session on basic ultrasound physics and knobology and were also provided with YouTube hyperlinks, and links to smart phone educational applications, which demonstrated a variety of bedside ultrasound techniques. In small group sessions, students learned how to evaluate patients for pathology associated with hypotension. A knowledge assessment questionnaire was administered at the end of the session and again 3 months later. Student knowledge was also assessed using different clinical scenarios with multiple-choice questions.

Results: One hundred and three 3rd year medical students participated in this study. Appropriate type of ultrasound was selected and accurate diagnosis was made in different hypotension clinical scenarios: pulmonary embolism, 81\% (95\% CI, 73\%-89\%); abdominal aortic aneurysm, $100 \%$; and pneumothorax, $89 \%$ (95\% CI, $82 \%-95 \%)$. The average confidence level in performing ultrasound-guided central line placement was $7 / 10$, focused assessment with sonography for trauma was $8 / 10$, inferior vena cava assessment was $8 / 10$, evaluation for abdominal aortic aneurysm was $8 / 10$, assessment for deep vein thrombus was $8 / 10$, and cardiac ultrasound for contractility and overall function was $7 / 10$. Student performance in the knowledge assessment portion of the questionnaire was an average of $74 \%(\mathrm{SD}=11 \%)$ at the end of workshop and $74 \%$ (SD $=12 \%) 3$ months later $(P=0.00)$.

Conclusion: At our institution, we successfully integrated ultrasound and critical problemsolving instruction, as part of a 1-day workshop for undergraduate medical education.

Keywords: point-of-care ultrasound, undergraduate medical education, protocol-driven education, problem-based learning

\section{Introduction}

Curriculum for undergraduate medical education is continuously analyzed with significant emphasis placed on the acquisition of knowledge and critical problem-solving skills. Team-based learning and case-based instruction have gained increasing popularity in recent years and have been adopted, in some format, by medical schools across the country based on the belief that these techniques will help foster the development of critical problem-solving skills. ${ }^{1,2}$ A study by Schmidmaier et al, ${ }^{3}$ has demonstrated that conceptual knowledge alone is insufficient for the successful application of critical 
problem-solving skills when making clinical decisions. In fact this study discovered that clinical experiences play a larger role in clinical decision making. ${ }^{3}$ Despite the merits of traditional undergraduate medical education as well as problem-based learning, research suggests that training using full-scale, high-fidelity simulation models is superior for the attainment of critical assessment and management skills. ${ }^{4,5}$

The benefits of sound critical problem-solving skills are most actualized when physicians face critically ill patients. Hypotensive patients, for example, frequently perplex clinicians, because the differential diagnosis of this patient population is both extensive and complex. Furthermore, the physical exam, the most easily accessible tool a physician can use, has been demonstrated to be inadequate to differentiate the etiology of hypotension. ${ }^{6,8}$ Another disconcerting aspect to this patient population is that hypotension carries high mortality rates (16\%-25\%). ${ }^{9}{ }^{10}$ In addition, 39\% of all deaths following traumatic injury are results of uncontrolled hemorrhage with unrecognized or inadequately treated hypotension. ${ }^{11}$

Early diagnosis and management of the hypotensive patient has been shown to decrease mortality and improve patient care, as a result it would seem prudent to develop high-fidelity learning models for the acquisition of the skills necessary to best care for these patients. ${ }^{12,13}$ Point-of-care ultrasound has been demonstrated to help clinicians narrow the differential diagnosis of the hypotensive patient. Furthermore, goal-directed ultrasound protocols have been implemented to reduce time to ultrasound evaluations, and these techniques are taught at many residency programs. ${ }^{10,12,14}$

Research in ultrasound education has confirmed that early exposure can improve ultrasound skills. ${ }^{15,16}$ As a result, medical schools have started to implement curricula to teach pointof-care ultrasound skills. ${ }^{17-22}$ Currently, the most commonly used sonographic protocol is the "extended focused assessment with sonography for trauma" (EFAST), and this protocol is taught during medical school at numerous institutions. ${ }^{5,18}$ Simulation training for undergraduate medical education has also demonstrated strengths over traditional education due to the combination of immediate feedback, repetitive practice, curriculum integration, and clinical relevance. ${ }^{23}$ To our knowledge, no prior study has incorporated the use of ultrasound to build critical problem-solving skills. The focus of this study was to evaluate a protocol-driven ultrasound education model for undergraduate medical education, with an emphasis on how the use of sonography in the evaluation of hypotension can help direct clinical problem-solving.

\section{Methods}

\section{Study design/setting/population}

This cross-sectional study was conducted at an academic medical center. This study was reviewed by the institutional review board of the University of Arizona and arbitrated as not systematic research. The institutional review board did not require informed consent from participants be obtained. The study participants were 3rd year medical students with minimal or no prior ultrasound training experience. Participation in the study was voluntary. Data were collected from September 2013 to March 2014.

\section{Study protocol}

At our institution, 3rd year medical students have periodic breaks from clinical rotations in which they meet as a group in the classroom setting. During this week, students are exposed to topics they will encounter in their clinical rotations, such as high-value health care, general procedures, pathology stations, and review of cardiopulmonary resuscitation. A 1-day educational workshop focusing on the use of point-of-care ultrasound for clinical problem-solving was integrated into one of these weeklong sessions. This ultrasound workshop was geared toward the use of "sonography in the evaluation of the hypotensive patient." Instructors for this course were department of emergency medicine faculty, fellows, and residents with expertise in point-of-care ultrasound. The components of ultrasound workshop included asynchronous learning, 1-hour didactic lecture, a hands-on workshop, and an assessment survey administered at the end of the workshop and at 3 months following the workshop.

Asynchronous learning assignments, which included reading materials, YouTube hyperlinks, and information regarding other ultrasound educational websites were provided to the students prior to the workshop. These educational tools describe or demonstrate the point-of-care ultrasound applications relevant to the evaluation of the hypotensive patient. Additionally, a 1-hour didactic lecture was provided to participating 3 rd year medical students. The purpose of this lecture was to review basic ultrasound physics, machine knobs/controls, and review the sonographic protocol for evaluation of hypotension. The hands-on skill sessions were developed based on recommendations made by the Council of Emergency Medicine Residency Directors and the Academy of Emergency Ultrasound regarding the competency skills required to assess the hypotensive patient. ${ }^{24}$ Trained human models were used at the skill stations. Instruction at skill stations included different components of sonographic protocol for the evaluation of the hypotensive patient (Table 1). During the cardiac station, 
Table I Skills station descriptions

\begin{tabular}{|c|c|}
\hline Sonographic skills station & Learning objectives and methods \\
\hline \multicolumn{2}{|l|}{ Sonography for hypotension } \\
\hline \multirow[t]{6}{*}{ Cardiac (30 minutes) } & How to assess the heart for activity and function. \\
\hline & Cardiac evaluation in a hypotensive patient can be life saving. In this station, students \\
\hline & learned how to obtain normal cardiac views. During this session, emphasis was placed on \\
\hline & right ventricle size, systolic versus diastolic collapse, presence of pericardial effusion, and \\
\hline & global function. Students learned which sonographic findings would be associated with \\
\hline & cardiac arrest, pulmonary embolism, and tamponade physiology. \\
\hline \multirow[t]{4}{*}{ Abdominal aorta (30 minutes) } & How to evaluate the abdominal aorta for aneurysms. \\
\hline & The proximal, mid, and distal aorta were evaluated in short and long axes. Anteroposterior \\
\hline & measurements of aorta were obtained in short axis. Students learned the sonographic \\
\hline & criteria (measurements) for the diagnosis of abdominal aortic aneurysm (AAA). \\
\hline EFAST (extended focused assessment with sonography & How to evaluate for intra-abdominal free fluid. \\
\hline \multirow[t]{5}{*}{ for trauma) examination (20 minutes) } & Right upper quadrant (RUQ): Morison's pouch/paracolic gutter; subxyphoid: pericardial \\
\hline & effusion and global cardiac function; left upper quadrant: free fluid above the spleen and \\
\hline & spleen-renal space; pelvis: free fluid adjacent to bladder in short and long axis; thorax: \\
\hline & pleural slide at bilateral anterior chest wall. Emphasis was placed on the RUQ when \\
\hline & limited by time. \\
\hline \multirow[t]{2}{*}{ EFAST individual challenge timed event ( 20 minutes) } & Evaluation of time to complete an EFAST exam. \\
\hline & Each student completed a supervised and timed EFAST exam (Maximum 3 minutes). \\
\hline \multirow[t]{3}{*}{ Inferior vena cava (IVC) evaluation (20 minutes) } & How to evaluate global volume status using IVC. \\
\hline & Emphasis of education was placed on plethoric IVC versus collapsible IVC as a measure \\
\hline & of pathology, such as tamponade, pulmonary embolism, and hypovolemia, respectively. \\
\hline \multirow[t]{6}{*}{ Lower extremity venous ultrasound (20 minutes) } & How to assess lower extremity veins for deep vein thrombus (DVT). \\
\hline & Students learned two-point compression lower extremity ultrasound. Emphasis placed \\
\hline & on common femoral vein and popliteal vein as per the 2008 JAMA article. ${ }^{26}$ Sonographic \\
\hline & findings associated with DVT were discussed. In addition, students were educated on the \\
\hline & need for anticoagulation in a patient with hypotension and DVT for presumed \\
\hline & pericardial effusion. \\
\hline \multirow[t]{5}{*}{ Ultrasound-guided central line placement (20 minutes) } & How to place a central venous catheter. \\
\hline & Students learned differences between large bore intravenous, triple lumen central venous \\
\hline & catheter, and cordis venous catheter. Emphasis was placed on needle guidance and \\
\hline & improvement of procedure through the use of ultrasound guidance (safety and success rates). \\
\hline & Evaluation of time to complete ultrasound-guided central line placement was performed. \\
\hline \multirow[t]{5}{*}{ Image review (20 minutes) } & How to recognize ultrasound pathology relevant to hypotension. \\
\hline & PowerPoint slideshow containing pathology relevant to the hypotensive patient. \\
\hline & The slide show contained images and videos of the following: positive peritoneal fluid \\
\hline & in all EFAST views, pleural effusion, cardiac tamponade, plethoric IVC, collapsible IVC, \\
\hline & hemothorax, pneumothorax, central line placement, and soft tissue foreign body. \\
\hline
\end{tabular}

students were taught to look for sonographic signs consistent with cardiogenic hypotension: decreased cardiac contractility in the case of decompensated heart failure; dilated right ventricle, decreased right ventricle free wall motion, and plethoric inferior vena cava (IVC) in the case of pulmonary embolism; and pericardial effusion in the case of cardiac tamponade.

At the end of the workshop, knowledge assessment was conducted using a 28-item questionnaire aimed to assess the following components: knowledge of the use of ultrasound for the evaluation of the hypotensive patient, self-assessed confidence of various ultrasound applications, opinions of the ultrasound workshop, opinions of protocol-based clinical problem solving, and evaluation of exposure to ultrasound.
In order to assess knowledge retention, a portion of the questionnaire was comprised of multiple-choice questions and clinical scenario questions. The multiple-choice questions assessed clinical management, sonographic findings, and basic ultrasound skills acquired during the workshop. The clinical scenario questions were two part questions that required students to choose the most appropriate diagnostic ultrasound application and to identify the pathology students were anticipating. Students were asked to assess their confidence in each of the sonographic skills that were taught during the workshop. The questionnaire was administered at the end of the workshop and 3 months following the workshop. 


\section{Data analysis}

All analyses were performed using Stata 11 (StataCorp LP, College Station, TX, USA). Data are presented as means and percentages with $95 \%$ confidence intervals. Continuous variables were compared between the two groups (post-test immediately after workshop versus 3-month post-test) using Student's $t$-test and Wilcoxon signed-rank test. The statistical level of significance was set at $P<0.05$.

\section{Results}

A total of 101 subjects participated in this ultrasound workshop, and $92 \%$ (93/101) completed the questionnaire at the end of the workshop. Forty-one percent of the students responded to our 3-month follow-up questionnaire. Only $17 \%$ (95\% CI, 9\%-25\%) of students had been taught how to use ultrasound to assess a patient with hypotension prior to the workshop. Ninety-one percent (95\% CI, $86 \%-97 \%$ ) had interest in learning ultrasound and would enroll in an optional ultrasound curriculum if given the opportunity. Ninety-six percent $(95 \%$ CI, $92 \%-100 \%)$ of students agreed that the use of "ultrasound protocol for the evaluation of the hypotensive patient" workshop helped them understand how ultrasound could be effectively integrated into clinical problem-solving. Similarly, 95\% (95\% CI, 90\%-100\%) felt that protocol-driven education was an effective teaching technique. Eighty percent (95\% CI, $72 \%-88 \%$ ) of students also felt that the ultrasound workshop helped them understand how ultrasound can change outcomes in acutely ill patients. After completing the ultrasound workshop, $100 \%$ of the medical students were able to complete the EFAST challenge within 3 minutes. Student performance in the knowledge assessment portion of the questionnaire was an average of $74 \%(\mathrm{SD}=11 \%)$ at the end of workshop and $74 \%(\mathrm{SD}=12 \%) 3$ months later $(P=0.00$; Figures 1 and 2). The confidence levels in each of the sonographic skills reported by students are summarized in Table 2.

\section{Discussion}

Our results suggest use of sonography for clinical problem solving such as in the evaluation of the hypotensive patient can be effectively taught to medical students. With increasing use of bedside sonography in the evaluation and management of critically ill patients, it is crucial for medical students to have ultrasound skills as they enter residency. To our knowledge, no prior studies have evaluated the integration of sonography into clinical problem solving sessions. We believe this approach generates more interest among medical students. Overall the students' response to the workshop was positive. Although the majority of students had not previously received ultrasound training, they were easily able to integrate its use in the evaluation of hypotension and quickly picked up on the skills required to scan.

This is the first ultrasound workshop designed to teach medical students the ultrasound applications used to evaluate hypotensive patients. Unlike traditional ultrasound education,

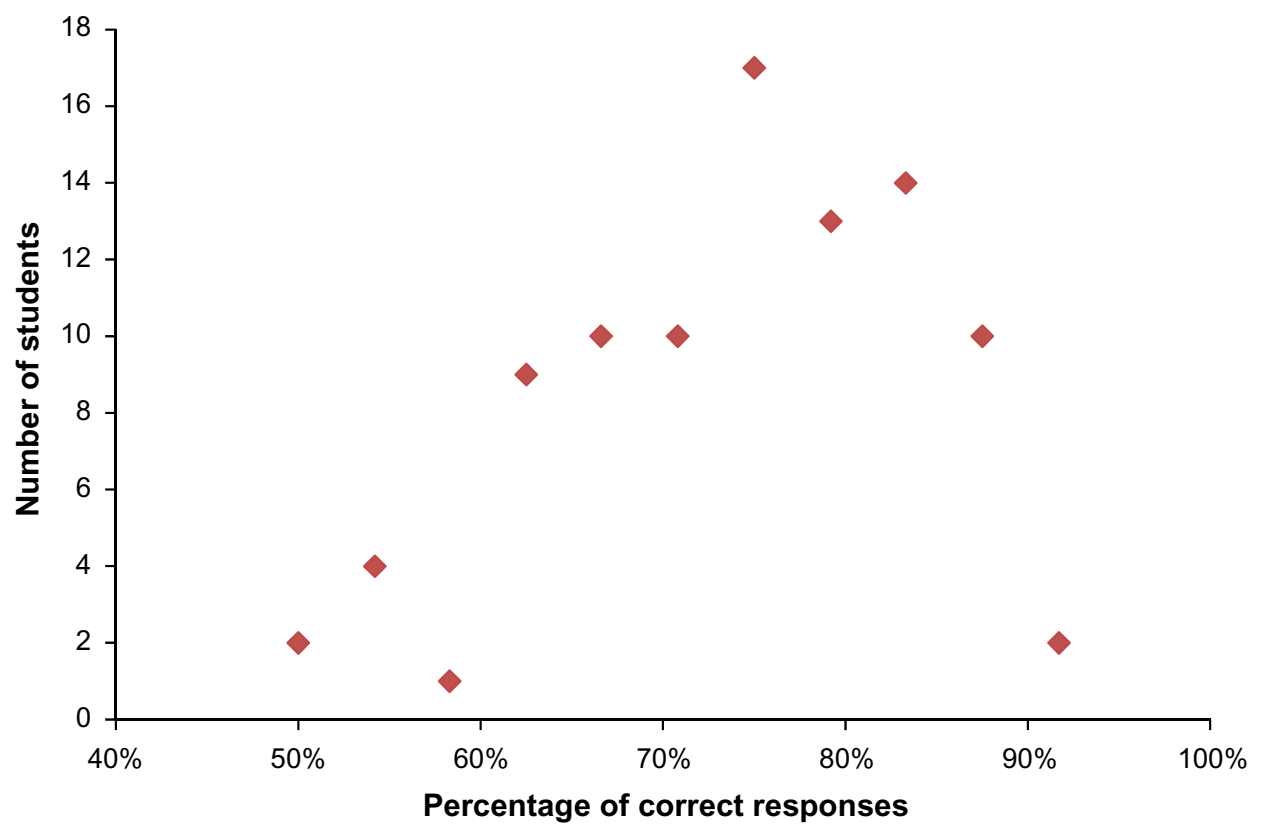

Figure I Student test scores immediately after the I-day workshop. 


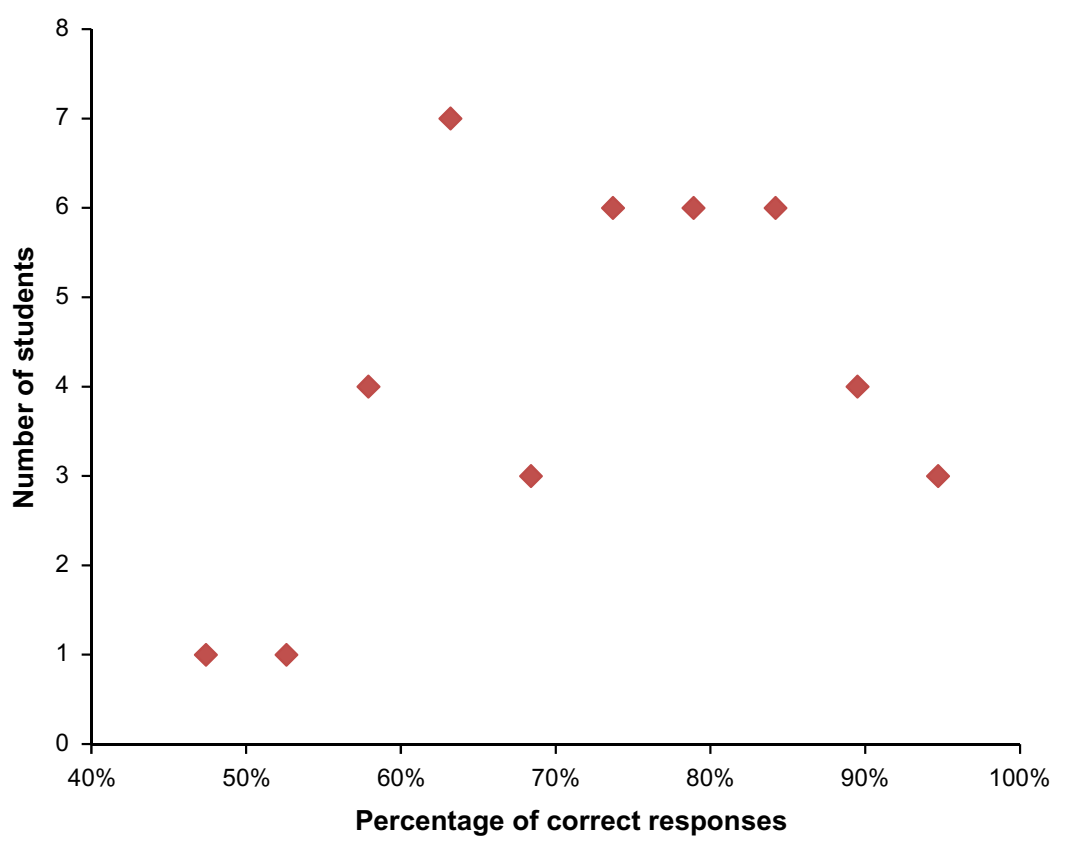

Figure 2 Student test scores 3 months after the I-day workshop.

which is organ based, our curriculum was protocol driven. As the students rotated through the stations within the ultrasound protocol for the hypotensive patient, they learned about the possible hypotensive pathologies associated with each ultrasound application. For example, when rotating through the Aorta station, students were taught that although an abdominal aortic aneurysm is enlarged at $3 \mathrm{~cm}$; in a hypotensive patient, an aneurysm greater than or equal to $6 \mathrm{~cm}$ is presumed ruptured. In addition, during the IVC station, students learned that IVC measurements are useful in extremes (flattened IVC = intravascular depletion and plethoric IVC $=$ heart failure/pericardial effusion).

Table 2 Students self-reported confidence with ultrasound skills on day I and day 90

\begin{tabular}{llll}
\hline Sonographic skill & $\begin{array}{l}\text { Confidence } \\
\text { on day I }\end{array}$ & $\begin{array}{l}\text { Confidence } \\
\text { on day 90 }\end{array}$ & $P$-value \\
\hline Performing EFAST exams & $7.82(\mathrm{SD}=1.7)$ & $5.34(\mathrm{SD}=2)$ & $P<0.05$ \\
Interpreting EFAST exams & $7.09(\mathrm{SD}=1.9)$ & $5.20(\mathrm{SD}=2.2)$ & $P<0.05$ \\
Volume assessment with IVC & $8.00(\mathrm{SD}=1.5)$ & $5.46(\mathrm{SD}=2.3)$ & $P<0.05$ \\
Evaluation for AAA & $8.06(\mathrm{SD}=1.6)$ & $5.59(\mathrm{SD}=2.5)$ & $P<0.05$ \\
$\begin{array}{l}\text { Assessment of cardiac } \\
\text { activity and function }\end{array}$ & $7.12(\mathrm{SD}=1.9)$ & $4.83(\mathrm{SD}=2.2)$ & $P<0.05$ \\
$\begin{array}{l}\text { Central line placement } \\
\text { Evaluation of lower }\end{array}$ & $6.99(\mathrm{SD}=1.9)$ & $4.37(\mathrm{SD}=2.1)$ & $P<0.05$ \\
extremities for DVT & $8.3 \mathrm{I}(\mathrm{SD}=1.5)$ & $5.56(\mathrm{SD}=2.2)$ & $P<0.05$ \\
\hline
\end{tabular}

Note: Confidence scores were measured from I-10: I was least confident and 10 was most confident.

Abbreviations: AAA, abdominal aortic aneurysms; DVT, deep vein thrombosis; EFAST, extended focused assessment with sonography for trauma; IVC, inferior vena cava.
Incorporating hands-on training sessions is paramount to teaching ultrasound as this requires spatial motor skills that have been shown to improve with experience. ${ }^{25}$ Our educational curriculum provided ample opportunities for students to gain hands-on experience with direct and immediate feedback from trained physicians. Based on previous experience, our goal was to create small groups with student to instructor ratios of $5: 1$. This increased the dedicated hands-on learning experience, and with minimal training students were able to acquire images for each of the stations. In fact, all students were able to complete an EFAST exam within the 3-minute timed station. Our results indicate that protocol-based ultrasound education was well received by the medical students. The ultrasound workshop created a comfortable learning environment in which students were able to learn diagnostic skills as well as procedural skills. In addition, critical thinking skills and conceptual knowledge was demonstrated on the post-test questionnaire. In fact, the average score on the questionnaire was $74 \%$ after the workshop despite the fact that the majority of students were ultrasound naïve.

Our study results indicate that students' knowledge was maintained beyond 3 months. However, the self-reported confidence in sonographic skills was significantly reduced (Table 2). It is possible that the students' lack of confidence is attributed to the lack of practice. In fact, $92 \%$ of the students had performed fewer than three ultrasounds in the preceding 3 months. Although the true impact of a 1-day workshop is 
unknown, students require practice in order to maintain and improve upon the spatial motor skills acquired during the workshop. In an era where ultrasound is easily accessible, developing an understanding of how to apply sonography to the bewildering patient will empower the next generation of critically thinking clinicians.

\section{Limitations}

Our study has several limitations including a small sample size. Although $92 \%$ of students responded to the questionnaire immediately after the 1-day event, only $41 \%$ of the students responded to the follow-up questionnaire 3 months after the event. Student knowledge retention was assessed, however, we did not test students' competency in acquiring ultrasound images. Another limitation of the study was that some of the results were based on student selfassessment. Furthermore, it is possible that students with greater interest in ultrasound participated in this study and in the follow-up questionnaire. One educational limitation was the number of ultrasound applications being taught during a 1-day workshop. These students were ultrasound naïve and although the students demonstrated comprehension and skill development, it was challenging and complex. Lastly, our study was not designed to assess overall impact of the ultrasound protocol nor was it designed to assess the impact of integrating ultrasound into critical problem-solving. Long-term sonographic skill retention, usage, and impact on patient care were not assessed.

\section{Conclusion}

At our institution, we successfully integrated ultrasound and critical problem solving instruction, as part of a 1-day workshop for undergraduate medical education.

\section{Acknowledgments}

We would like to thank Mindray Medical International (Shenzhen, People's Republic of China) for providing ultrasound machines for this event.

\section{Disclosure}

The authors report no conflicts of interest in this work.

\section{References}

1. Burgess AW, McGregor DM, Mellis CM. Applying established guidelines to team-based learning programs in medical schools: a systematic review. Acad Med. 2014;89:678-688.

2. Amini R, Stolz LA, Gross A, et al. Theme-based teaching of point-ofcare ultrasound in undergraduate medical education. Intern Emerg Med. 2015;10(5):613-618.
3. Schmidmaier R, Eiber S, Ebersbach R, et al. Learning the facts in medical school is not enough: which factors predict successful application of procedural knowledge in a laboratory setting? BMC Med Educ. 2013;13:28.

4. Steadman RH, Coates WC, Huang YM, et al. Simulation-based training is superior to problem-based learning for the acquisition of critical assessment and management skills. Crit Care Med. 2006;34:151-157.

5. Amini R, Kartchner JZ, Stolz LA, Biffar D, Hamilton AJ, Adhikari S. A novel and inexpensive ballistic gel phantom for ultrasound training. World J Emerg Med. 2015;6(3):225-228.

6. McGee S, Abernethy WB, Simel DL. The rational clinical examination. Is this patient hypovolemic? JAMA. 1999;281:1022-1029.

7. Wo CC, Shoemaker WC, Appel PL, Bishop MH, Kram HB, Hardin E. Unreliability of blood pressure and heart rate to evaluate cardiac output in emergency resuscitation and critical illness. Crit Care Med. $1993 ; 21: 218-223$

8. Moore CL, Rose GA, Tayal VS, Sullivan DM, Arrowood JA, Kline JA. Determination of left ventricular function by emergency physician echocardiography of hypotensive patients. Acad Emerg Med. 2002;9: 186-193.

9. Jones AE, Stiell IG, Nesbitt LP, et al. Nontraumatic out-of-hospital hypotension predicts inhospital mortality. Ann Emerg Med. 2004;43: 106-113.

10. Jones AE, Tayal VS, Sullivan DM, Kline JA. Randomized, controlled trial of immediate versus delayed goal-directed ultrasound to identify the cause of nontraumatic hypotension in emergency department patients. Crit Care Med. 2004;32:1703-1708.

11. Sauaia A, Moore FA, Moore EE, et al. Epidemiology of trauma deaths: a reassessment. J Trauma. 1995;38:185-193.

12. Perera P, Mailhot T, Riley D, Mandavia D. The RUSH exam: Rapid Ultrasound in SHock in the evaluation of the critically 11l. Emerg Med Clin North Am. 2010;28:29-56, vii.

13. Volpicelli G, Lamorte A, Tullio M, et al. Point-of-care multiorgan ultrasonography for the evaluation of undifferentiated hypotension in the emergency department. Intensive Care Med. 2013;39: 1290-1298.

14. Amini R, Adhikari S, Fiorello A. Ultrasound competency assessment in emergency medicine residency programs. Acad Emerg Med. 2014;21(7):799-801.

15. Butter J, Grant TH, Egan M, et al. Does ultrasound training boost Year 1 medical student competence and confidence when learning abdominal examination? Med Educ. 2007;41:843-848.

16. Kobal SL, Trento L, Baharami S, et al. Comparison of effectiveness of hand-carried ultrasound to bedside cardiovascular physical examination. Am J Cardiol. 2005;96:1002-1006.

17. Hoppmann R, Cook T, Hunt P, et al. Ultrasound in medical education: a vertical curriculum at the University of South Carolina School of Medicine. J S C Med Assoc. 2006;102:330-334.

18. Rao S, van Holsbeeck L, Musial JL, et al. A pilot study of comprehensive ultrasound education at the Wayne State University School of Medicine: a pioneer year review. J Ultrasound Med. 2008;27:745-749.

19. Bahner DP, Royall NA. Advanced ultrasound training for fourth-year medical students: a novel training program at The Ohio State University College of Medicine. Acad Med. 2013;88:206-213.

20. Bahner DP, Jasne A, Boore S, Mueller A, Cortez E. The ultrasound challenge: a novel approach to medical student ultrasound education. J Ultrasound Med. 2012;31:2013-2016.

21. Dreher SM, DePhilip R, Bahner D. Ultrasound exposure during gross anatomy. J Emerg Med. 2014;46:231-240.

22. Hoyer R, Means R, Robertson J, et al. Ultrasound-guided procedures in medical education: a fresh look at cadavers. Intern Emerg Med. Epub 2015 Aug 15.

23. Issenberg SB, McGaghie WC, Petrusa ER, Lee Gordon D, Scalese RJ. Features and uses of high-fidelity medical simulations that lead to effective learning: a BEME systematic review. Med Teach. 2005;27: $10-28$. 
24. Lewiss RE, Pearl M, Nomura JT, et al. CORD-AEUS: consensus document for the emergency ultrasound milestone project. Acad Emerg Med. 2013;20:740-745.

25. Nicholls D, Sweet L, Hyett J. Psychomotor skills in medical ultrasound imaging: an analysis of the core skill set. J Ultrasound Med. 2014;33: 1349-1352.
26. Bernardi E, Camporese G, Büller HR, et al. Serial 2-point ultrasonography plus D-dimer vs whole-leg color-coded Doppler ultrasonography for diagnosing suspected symptomatic deep vein thrombosis: a randomized controlled trial. JAMA. 2008;300:1653-1659.

\section{Publish your work in this journal}

Advances in Medical Education and Practice is an international, peerreviewed, open access journal that aims to present and publish research on Medical Education covering medical, dental, nursing and allied health care professional education. The journal covers undergraduate education, postgraduate training and continuing medical education including emerging trends and innovative models linking education, research, and health care services. The manuscript management system is completely online and includes a very quick and fair peer-review system. Visit http://www.dovepress.com/testimonials.php to read real quotes from published authors.

Submit your manuscript here: http://www.dovepress.com/advances-in-medical-education-and-practice-journal 